\title{
Design Foundations for AI Assisted Decision-Making: A Self Determination Theory Approach
}

\author{
Triparna de Vreede, Mukhunth Raghavan, Gert-Jan de Vreede \\ University of South Florida \\ tdevreede@usf.edu, mukhunth@usf.edu, gdevreede@usf.edu
}

\begin{abstract}
Progress of technology and processing power has enabled the advent of sophisticated technology including Artificial Intelligence (AI) agents. AI agents have penetrated society in many forms including conversation agents or chatbots. As these chatbots have a social component to them, is it critical to evaluate the social aspects of their design and its impact on user outcomes. This study employs Social Determination Theory to examine the effect of the three motivational needs on user interaction outcome variables of a decision-making chatbot. Specifically, this study looks at the influence of relatedness, competency, and autonomy on user satisfaction, engagement, decision efficiency, and decision accuracy. A carefully designed experiment revealed that all three needs are important for user satisfaction and engagement while competency and autonomy is associated with decision accuracy. These findings highlight the importance of considering psychological constructs during AI design. Our findings also offer useful implications for AI designers and organizations that plan on using AI assisted chatbots to improve decision-making efforts.
\end{abstract}

\section{Introduction}

The past two decades have seen a tectonic shift in the manner in which technology has evolved and integrated into human lives. Technology is getting increasingly sophisticated and we are now able to process more data faster than ever before [40]. This exponential increase in computing power along with strides made in the machine learning has enabled Artificial Intelligence (AI) to become a ubiquitous companion in modern society.

However, the integration of AI has not been without its challenges. For instance, the hidden biases that were introduced in Amazon hiring algorithms resulted in prejudice against women and minorities [34]. Similarly, a lack of evolved understanding of the autonomous driving algorithm resulted in an Uber car killing a pedestrian [70]. These unwelcome outcomes were the result of an incomplete alignment of human characteristics with the $\mathrm{AI}$ design resulting in gaps in $\mathrm{AI}$ usability. To utilize AI most efficiently, it is important to examine and understand the effect of $\mathrm{AI}$ interactions on human behavior. AI performance and design is one of the critical aspects of such an understanding.
Compared to other sophisticated machines, AI typically has greater anthropomorphic characteristics [15]. This key difference between AI and other forms of IT makes it important for user interaction design to be more anticipatory of human reactions and ensuing outcomes. AI needs to be designed with a holistic focus on creating optimal interactions between human and machine. When AI design does not accurately anticipate accommodating the nature of human interaction, the resultant outcomes may be less than optimal. In other words, given the complexities and intricacies of an AI artifact, design considerations cannot exist in vacuum. Developers need to be knowledgeable about the human, psychological, and ethical considerations prior to designing an $\mathrm{AI}$ artifact since each AI design consideration may result in a different outcome when the AI is put to use. Different instantiations of a design may lead to very different results and developers have to keep the desired outcome in mind before designing the AI. Therefore, it is critical that these development efforts are bolstered by AI design research such that developers have a better understanding of the implications of their design choices. AI design research is not only important for AI efficiency and effectiveness but is also critical to managing the long-term effects of AI use on the well-being of users. With an increased understanding of the relationship between designs and outcomes based on theory and past research, developers can design appropriate AI for current user needs without causing inadvertent harm.

In order to establish AI design guidelines, it is important to lay a foundation that explains why those design guidelines matter. One way to contribute to this foundation is by exploring various factors that are expected to influence human-AI interaction and human behavior with AI. To guide such exploration, we use a specific theoretical lens: Self Determination Theory (SDT). Our current study uses SDT as a foundation to examine the effect of competency, relatedness, and autonomy on AI performance-related measures like outcome quality and speed along with well-being related measures like satisfaction and engagement. We expect that the results of our study will inform AI designers and developers to better manage AI design features depending on the outcome desired.

Our paper is structured as follows. In the next section, we provide an overview of SDT in the context of AI research and examine the relationship between three 
needs (competency, relatedness, and autonomy) and satisfaction, engagement, speed of decision-making, and accuracy of decision-making. Next, we describe our method and results. We conclude with a discussion of implications, limitations, and future research directions.

\section{Background and Research Model}

One advantage of combining AI with big data analytics is the ability to extract insights to make better decisions. Evidence of such benefits are already being documented in areas like healthcare [4], consumer behavior [39], military [49], and surveillance [73]. For instance, trading bots and robo-advisors regularly aid investor decision-making [57, 60]. AI decision-making also is used to improve efficiency of financial institutions through fraud detection and asset management [18]. Studies in healthcare have shown how AI assisted decision-making has played a significant role in cancer detection and diagnosis [17] [35]. In addition to helping with diagnoses, AI applications also aid in reducing hospital inefficiencies and reducing medical based errors [48] [5]. AI assisted decision-making has also been integrated with the service industry and is helping engage customers while providing various service benefits that range from helping with analytical tasks to providing empathetic responses [26] [27].

Huang and Rust [26] point out that AI intelligence can be categorized into four intelligences, namely mechanical, analytical, intuitive, and empathetic. Mechanical intelligence is the ability of AI to perform routine, repeated tasks that do not require much learning or novel responses. The AI agent need not learn from its previous experiences in order to successfully perform these tasks in the future. Analytical intelligence is the ability of AI to process information for the purpose of learning and problem solving $[64,65]$. This intelligence involves information processing, logical reasoning, and mathematical skills [64]. Intuitive intelligence is the ability of AI to adjust effectively to novel situations based on previous learning. This intelligence is derived from the holistic integration of previous learning and application to novel situations [64]. Empathetic intelligence is the ability of AI to recognize and understand user emotions and respond appropriately to them [20] [31]. AI agents should be designed in a manner that incorporates varying degrees of each type of intelligence depending on the nature of the decisionmaking assistance that is required by the user. AI agents can take various forms and can be embodied in the form of robots or can be disembodied in the form of voice activated agents [63]. Chatbots are currently the most common form of AI agents being used by organizations $[2,19,55]$ and they are the focus of our study.
Chatbots are conversational AI agents that interact with users using natural language [58]. They are capable of holding conversations relevant to the chatbot's programming from the users' voice or text command. The origin of chatbots goes back to 1950 [69]. Some of the first chatbot technologies that mimicked human conversations were ELIZA [72] and ALICE [71]. We define a chatbot as "disembodied conversational agent that can hold a natural language conversation via textbased environment to either engage the user in a general-purpose or task-oriented conversation" [10].

Chatbot technologies have taken off at a rapid pace in recent years and have permeated almost every walk of life from customer service [68] to job screening [8] . For instance, in 2016 Microsoft presented its vision of "conversation as a platform" and began incorporating chatbots in most of its applications like Skype and MS Office. Facebook followed suit with incorporating chatbots on Messenger. Other social media platforms like Reddit also use chatbots to interact with the users. On the business side, chatbots are now an essential feature for customer service as well as gathering user feedback [24]. In recent years, the role of chatbots has steadily progressed from information collection and dissemination [46] to being recommendation agents [1] and assisting in decision-making [43]. Advances in graphic interface design have allowed designers to incorporate substantial visual mechanisms to create an interactive experience between AI and users [75] . While the effect of these design features has been examined for other types of AI agents, there appears to be few research studies that explore features needed to create efficient and effective chatbots to aid in decisionmaking. Consequently, it may be useful to step back and evaluate the design features that are relevant to optimal user outcomes in a chatbot experience in general and in decision-making scenarios in particular.

\subsection{Self-Determination Theory}

In this paper we focus on chatbot design features and their impact on user interaction through the lens of Self Determination Theory (SDT). SDT is a macro theory of motivation [54]. It represents a framework that assumes that people are curious by nature, seek coherence, and inherently enjoy being productive [13, 54]. It examines the motivations behind individual decisions and evaluates the conditions under which people feel motivated and engaged to perform their tasks successfully.

SDT identifies three basic needs, which are competence, relatedness, and autonomy. These needs, if satisfied, allow for personal growth, enjoyment of effort, mastering of challenges, and integrating new experiences. Competence refers to the need to feel 
confident about the ability to effectively engage with one's own environment and achieve desired outcomes. Relatedness refers to an individual's need to establish close relationships and identify with others. Autonomy refers to the need to be in control of one's behavior such that the choices that are being made arise from the self rather than from an external or a forced circumstance. These three psychological needs are interrelated and complementary to each other, yet they can exist independently and have a different effect on human behavioral outcomes [54]. According to SDT, the conditions that support an individual's experience of autonomy, competence, and relatedness foster most volitional and high-quality outcomes while thwarting these needs results in a detrimental impact on the quality of outcomes as well as the well-being of the individual.

SDT is a well-received concept in the IS literature. Researchers have studied SDT in contexts such as technology learning [52] [61], gaming [44], information security [41], and robotics [25]. For instance, Sørebø and colleagues [61] found that the three needs were predictive in determining the perceived usefulness of an IS artifact. Similarly, [41] observed that security messages that appeal to an individual's motivational needs are more likely to elicit secure responses than messages that elicit fear. Yet, to date, there is only limited research that has specifically looked into the effect of SDT on the chatbot design $[9,45]$. For instance, Chaves and colleagues [9] utilized a meta-analytical method to examine how social characteristics of a conversational chatbot can benefit user interactions with the chatbot. Nguyen et al. [45] used SDT to study the differences in satisfaction with a website and with a chatbot. The goal of our study is to further examine the impact of SDT on various user outcomes that determine the usefulness of the chatbot assistant. Specifically, we evaluate the effect of three motivational needs on outcomes in a decision-making context such as satisfaction, engagement, decision-making efficiency, and decision-making accuracy.

\subsection{SDT and Satisfaction}

We propose that the three motivational needs within SDT that a user feels regarding a chatbot are predictive of their perceived satisfaction towards the chatbot. Despite the ubiquity of chatbots in modern society, the design of chatbots often fails to meet users' expectations [29] [38] [74]. One of the manifestations of such failing expectations is the drop in the satisfaction levels with the chatbot experience. We define satisfaction in this study as an "affective arousal towards an object related to some state or outcome desired by an individual" [7].

Literature supports the view that design considerations that do not meet the intrinsic motivational needs of users fail to satisfy them. For example, Nguyen \& Sidorova [45] examined user interactions with a chatbot using the SDT approach. They found that perceived competency, relatedness, and autonomy were all related to satisfaction with their own performance as well as their satisfaction with the system. Similarly, Rezvani and colleagues [51] employed SDT to show that transformational and transactional leadership styles affect users' motivational needs, which in turn impacts ERP continuance intentions as reflected by the user satisfaction and perceived usefulness of the system.

Within the chatbot literature, studies suggest that chatbot's interactional goals should include social capabilities along with functional performance [29] [37]. Studies show that chatbots that do not meet users' relatedness needs often result in user dissatisfaction and frustration [38] [74]. Other studies show that autonomy and competence also are important factors in determining the satisfaction with the chatbots [50]. Therefore, we hypothesize that:

H1: Perceived autonomy regarding the chatbot influences perceived satisfaction with the chatbot, such that as perceived autonomy over the chatbot increases, satisfaction with the chatbot increases.

H2: Perceived competency regarding the chatbot influences the perceived satisfaction with the chatbot, such that as perceived competency with using the chatbot increases, satisfaction with the chatbot experience increases.

H3: Perceived relatedness regarding the chatbot influences the perceived satisfaction with the chatbot, such that as perceived relatedness with the chatbot increases, satisfaction with the chatbot experience increases.

\subsection{Engagement and Satisfaction}

We propose that the degree of satisfaction that a chatbot user derives from the use of the chatbot is predictive of the degree of engagement experienced with the chatbot. In our study, we follow de Vreede et al. [12], who define engagements a three-part phenomenon:

1. Affective/Emotional engagement: the extent to which individuals experience a positive psychological reaction or attachment towards a specific activity or situation.

2. Behavioral engagement: the extent to which the individuals can be observed to exert effort and show persistence to remain involved in an activity or situation.

3. Cognitive engagement: the extent to which individuals are cognitively absorbed in a task or activity resulting in a reduced awareness of their surroundings. 
In addition, we propose that satisfaction plays a mediating role in the relationship between the three motivational needs of the chatbot users and their degree of engagement with the chatbot.

This is based on research evidence regarding the positive effects of satisfaction with IS on user engagement. For instance, studies on mobile technology adoption show that satisfaction with the technology is predictive of continued engagement with the mobile phones [32]. Gamification research shows that satisfaction mediates the relationship between game dynamics and user engagement and enjoyment [66]. Other studies show that users that are deeply engaged in an information seeking activity with a system experience greater satisfaction with that system [56]. While there is limited research on the effect of satisfaction with a chatbot on the degree of engagement with chatbots, the extant research shows that satisfaction that users feel with personalization of the chatbot is predictive of their engagement with the chatbot [16]. Therefore, we hypothesize that:

H4: Perceived satisfaction with the chatbot influences engagement with the chatbot, such that as perceived satisfaction with the chatbot increases, engagement also increases.

\subsection{SDT and Decision-Making Efficiency}

We propose that the degree to which some of the motivational needs of SDT are fulfilled by the AI assisted chatbot influence decision-making efficiency. That is, perception of competency and perception of autonomy impacts the efficiency of decision-making by a chatbot user. Specifically, we propose that sense of competency about using the chatbot increases the decision-making efficiency and the sense of autonomy reduces that efficiency.

Evidence of effect of perception of competence on decision-making speed can be found in the literature. While we could not find a direct assessment of competence, self-efficacy is a similar construct as perception of competency and thus has been used as a proxy for competence. In their study, Hepler and Feltz [22], found that students with higher perception decision-making self-efficacy were speedier in making the decisions as well. Yet another study showed that higher self-efficacy was a significant and consistent predictor of decision-making speed in sports [23]. In the information systems context, studies show that decision makers with a higher computer self-efficacy made faster decisions when working on IS tasks [14] [28].

There is limited evidence regarding the effect of autonomy on decision-making speed. However, the extant literature appears to point towards a mixed relationship between choice abundance and decision- making speed. For instance, studies show that availability of choice and autonomy to pick between options may be beneficial for decision efficiency but the information overload can mitigate those effects [62].

Therefore, we propose that:

H5: Perceived competency with the chatbot influences the efficiency of decision-making, such that as perceived competency with the chatbot increases, decision-making efficiency also increases.

H6: Perceived autonomy with the chatbot influences the efficiency of decision-making, such that as perceived autonomy with the chatbot increases, decision-making efficiency decreases.

\subsection{SDT and Decision-Making Accuracy}

Finally, we propose that degree to which some of the motivational needs of SDT are fulfilled by the AI assisted chatbot also influence the accuracy of the decisionmaking. That is, perception of competency and perception of autonomy impacts the accuracy of the decision made by a chatbot user. Specifically, we propose that the sense of competency about using the chatbot leads to an increase in decision accuracy while the sense of autonomy decreases the accuracy of the decision.

Exploration of past research on the relationship between competence and decision-making accuracy reveals mixed outcomes [21, 30, 47]. For example, one study found that individuals who scored low in decisionmaking competence were unable to make optimal decisions [47]. However, results from another study showed that higher decision-making self-efficacy did not predict decision-making performance [21]. Yet another study that compared physicians' self-evaluation of their decision-making competence and performance yielded mixed results with $65 \%$ of those surveyed demonstrating little, no, or an inverse relationship between self-evaluation of competence and actual competence, with accuracy being worst among the physicians who had the greatest confidence or lowest skill [11]. However, when an AI chatbot is used to help with decision-making, we argue that the sense of competency that the chatbot provides improves the selfefficacy of the user regarding the decision-making scenario. This sense of self-efficacy in turn will help the user reach decisions faster [23]. Therefore, in the case of an AI assisted chatbot, we contend that there will be an improvement in decision-making efficiency when the sense of competency is enhanced.

Literature on the effect of autonomy on decisionmaking accuracy supports a negative relationship. For instance, in a consumer research study it was observed that audiences who had an abundance of television channels to choose from made less than optimal decisions regarding the television shows to watch and ended up 
watching same genres and choosing fewer channels [3]. Another study showed that high choice abundance creates memory confusion issues resulting in less than optimal decision-making [36]. Therefore, we propose that:

H7: Perceived competency with the chatbot influences the accuracy of decision-making, such that as perceived competency with the chatbot increases, decision-making accuracy also increases.

H8: Perceived autonomy with the chatbot influences the accuracy of decision-making, such that as perceived autonomy with the chatbot increases, decision-making accuracy decreases.

Figure 1 shows the proposed model and hypotheses.

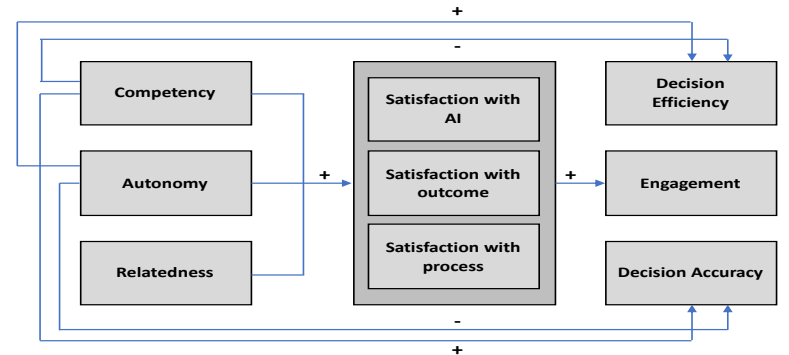

Figure 1. Research Model.

\section{Method}

\subsection{Participants}

Our sample consisted of 423 undergraduate students recruited from a South Eastern US university. Participants ranged in age from 17 to 50 years $(M=20.7$, $S D=3.4)$ and identified mostly as male $(51.3 \%)$. The racial breakdown of the sample was: $61.7 \%$ White, 15.8\% Asian, 8.3\% African American, 0.2\% Native American, $0.2 \%$ Pacific Islander, and 13.2\% Other (e.g., biracial, or preferred not to disclose race).

\subsection{Procedure}

This study utilized a $2 \times 2 \times 2$ between-subjects design (autonomy $\mathrm{x}$ relatedness $\mathrm{x}$ competency) with each participant randomly assigned to one of eight conditions where the perceived level of each of the three SDT needs was manipulated to elicit high or low levels.

Participants were presented with a complex decision-making task in which they were asked to take on the role of a project manager. In the scenario, participants were to solve a logic puzzle and assign the correct role and number of hours to each of four employees of an organizational team project. In this task, there was a total of eight possible correct responses that participants could provide.

Participants were offered support from a virtual assistant chatbot. The chatbot was designed to provide pertinent information via messages based on prompts that the participant responded to. A Logigram puzzle with an interactive $3 \times 4$ logic grid was provided to help participants sort each individual into the appropriate roles and number of work hours. Instructions were provided along with an example of the task completion before participants were taken to the actual task.

All participants received the same amount of information and all the details required to complete the task successfully. However, the interactions between the participants and the chatbot differed across each of the eight conditions to elicit perceptions of high or low autonomy, relatedness, and competency.

In the high autonomy conditions, the participant had the opportunity to request additional information when needed. The chatbot prompted participants by asking if they would like additional information and what category of information they would like to receive. In the low autonomy conditions, all required information was provided automatically without allowing the participant to request additional information.

In the high relatedness conditions, the chatbot introduced itself by name and asked the participant for a name. Throughout the conversation, the bot used the participant's name and referred to itself as "I". An avatar was also displayed by its messages. In the low relatedness conditions, the chatbot neither introduced itself nor asked for the participant's name. It was also designed to not refer directly to the participant throughout the task. The participant simply received relevant instructions and information, rather than conversing with the bot.

In the high competency conditions, participants were provided with the opportunity to use an interactive logic grid to complete a practice puzzle along with a thorough explanation of the task before being taken to the actual task. In the low competency conditions, participants were not provided a practice grid but were simply shown images of a logic grid being completed along with the explanations on how to use the grid to solve the puzzle.

\subsection{Measures}

Decision Accuracy and Efficiency. Accuracy of task completion was assessed by the number of correct decisions made at the end of the task. Efficiency of task completion was assessed by the amount of time taken to make all decisions and complete the task such that greater time taken is regarded as lower efficiency.

Manipulation check. An adaptation of the 9-item self-report scale [33] was used as a check of the manipulation of the perceived SDT needs. This scale included four items assessing participants' perceived level of autonomy, four items assessing perceived relatedness, and a single item assessing perceived 
competency. An example item from the Relatedness subscale reads: "I could relate to the AI." For the Autonomy and Relatedness subscales, respondents were asked to indicate the degree to which they agree with each item on a seven-point Likert-type scale. The single item assessing competency asked respondents to rate how competent they felt when solving the problem after receiving training. The full scale and two subscales demonstrated good internal consistency in the present sample (full scale: $\alpha=.86$; Autonomy: $\alpha=.85$; Relatedness: $\alpha=.89$ ).

Satisfaction. A 15-item self-report scale was used to measure participants' satisfaction with the chatbot interaction. The scale is based on [6] and contains three subscales that assess satisfaction with the AI, satisfaction with the process, and satisfaction with the outcome. An example item from the Satisfaction with the AI subscale reads: "I liked having the AI to help me with the problemsolving exercise." Respondents were asked to indicate the degree to which they agree with each item on a sevenpoint Likert-type scale. The full scale and all three subscales demonstrated high internal consistency in the present sample (full scale: $\alpha=.96$; Satisfaction with the AI: $\alpha=.95$; Satisfaction with the Process: $\alpha=.96$; Satisfaction with the Outcome: $\alpha=.97$ ).

Engagement. A 13-item self-report scale was used to measure participants' engagement with the chatbot interaction. The scale is adapted from [12] and contains three subscales that assess cognitive, behavioral, and emotional engagement. An example item from the Behavioral Engagement subscale reads: "I dutifully followed the instructions for solving the problem." Respondents were asked to indicate the degree to which they agree with each item on a seven-point Likert-type scale. The full scale and all three subscales demonstrated good internal consistency in the present sample (full scale: $\alpha=.95$; Cognitive Engagement: $\alpha=$ .82; Behavioral Engagement: $\alpha=.95$; Emotional Engagement: $\alpha=.92$ ).

\subsection{Data Analysis}

The data were first screened for potential issues with multicollinearity and normality. In the current study, the variables were not distributed normally, however, there was no issue with multicollinearity. Following this, a structural equation model (SEM) was analyzed to examine the direct, interactive, and mediated effects of the variables of interest using the diagonally weighted least squares (DWLS) method which is robust to missing data and data nonnormality [42]. Fit indices including Chi squared, the comparative fit index (CFI), root mean square error of approximation (RMSEA), and the standardized root mean square residual (SRMR) were used to assess model fit. All analyses were conducted on R [67] and SEM analyses were conducted using the lavaan package [53].

\section{Results}

Descriptive statistics and correlations for the observed and latent variables are presented in Table 1. Figure 2 shows the SDT needs in each of the conditions (the three letter code represents levels of autonomy, relatedness, and competency). The figure shows that participants differentially perceived SDT needs according to the condition. For example, in conditions that were designed to elicit feelings of high relatedness (such as HHL, middle frame, first row), perceived relatedness was higher than in conditions that were designed to elicit feelings of low relatedness (such as LLH, left frame, third row). These differences were supported by the subsequent tests of the difference in means. The chatbot interaction was able to successfully manipulate participants' perceived levels of autonomy, $t(420.03)=-3.82, p$ $<0.01,95 \% \mathrm{CI}=[-0.89,-0.28]$, and relatedness, $t(420.65)$ $=4.90, p<0.01,95 \% \mathrm{CI}=[0.37,0.87]$. However, the manipulation check revealed that competency was not successfully manipulated by the chatbot interaction, $t(418.57)=0.16, p=0.87,95 \% \mathrm{CI}=[-0.21,0.24]$.

Table 1: Means, standard deviations, and correlations.

\begin{tabular}{|c|c|c|c|c|c|c|c|c|c|}
\hline Variable & $M$ & $S D$ & 1 & 2 & 3 & 4 & 5 & 6 & 7 \\
\hline 1. Time & 12.99 & 14.07 & & & & & & & \\
\hline 2. Accuracy & 6.01 & 2.23 & $.33^{* *}$ & & & & & & \\
\hline 3. Relatedness & 4.55 & 1.34 & .07 & .06 & & & & & \\
\hline 4. Autonomy & 4.41 & 1.60 & $.10^{*}$ & .03 & $.53^{* *}$ & & & & \\
\hline 5. Competency & 2.47 & 1.17 & $.14^{* *}$ & $.36 * *$ & .09 & .02 & & & \\
\hline 6. Self-efficacy & 3.12 & 0.62 & $.13^{* *}$ & $.19 * *$ & -.03 & .02 & $.20^{* *}$ & & \\
\hline 7. Satisfaction & 5.24 & 1.26 & $.13^{* *}$ & $.31^{* *}$ & $.49^{* *}$ & $.40^{* *}$ & $.27^{* *}$ & $.16^{* *}$ & \\
\hline 8. Engagement & 5.46 & 1.17 & $.21 * *$ & $.39 * *$ & $.40^{* *}$ & $.36 * *$ & $.33^{* *}$ & $.21^{* *}$ & $.66^{* *}$ \\
\hline
\end{tabular}

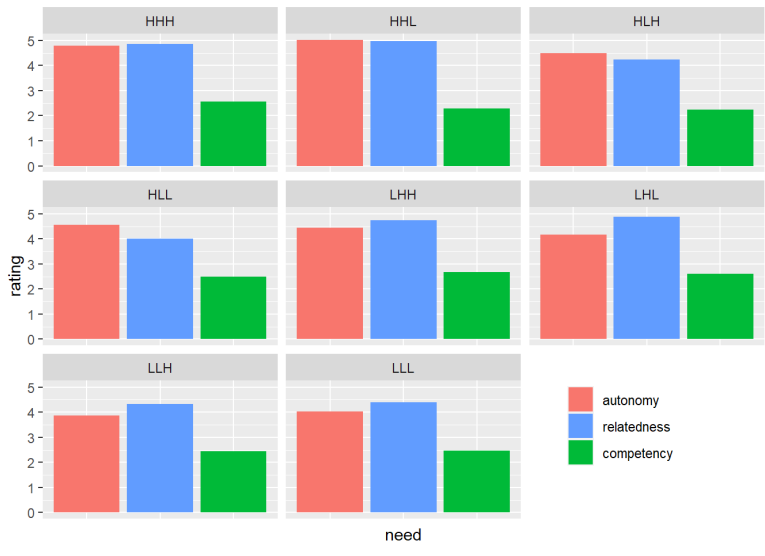

Figure 2: Perceived SDT needs in the eight conditions.

Figure 3 presents the relationships between the variables considered in the current study and their path coefficients. The data showed adequate fit to the model, 


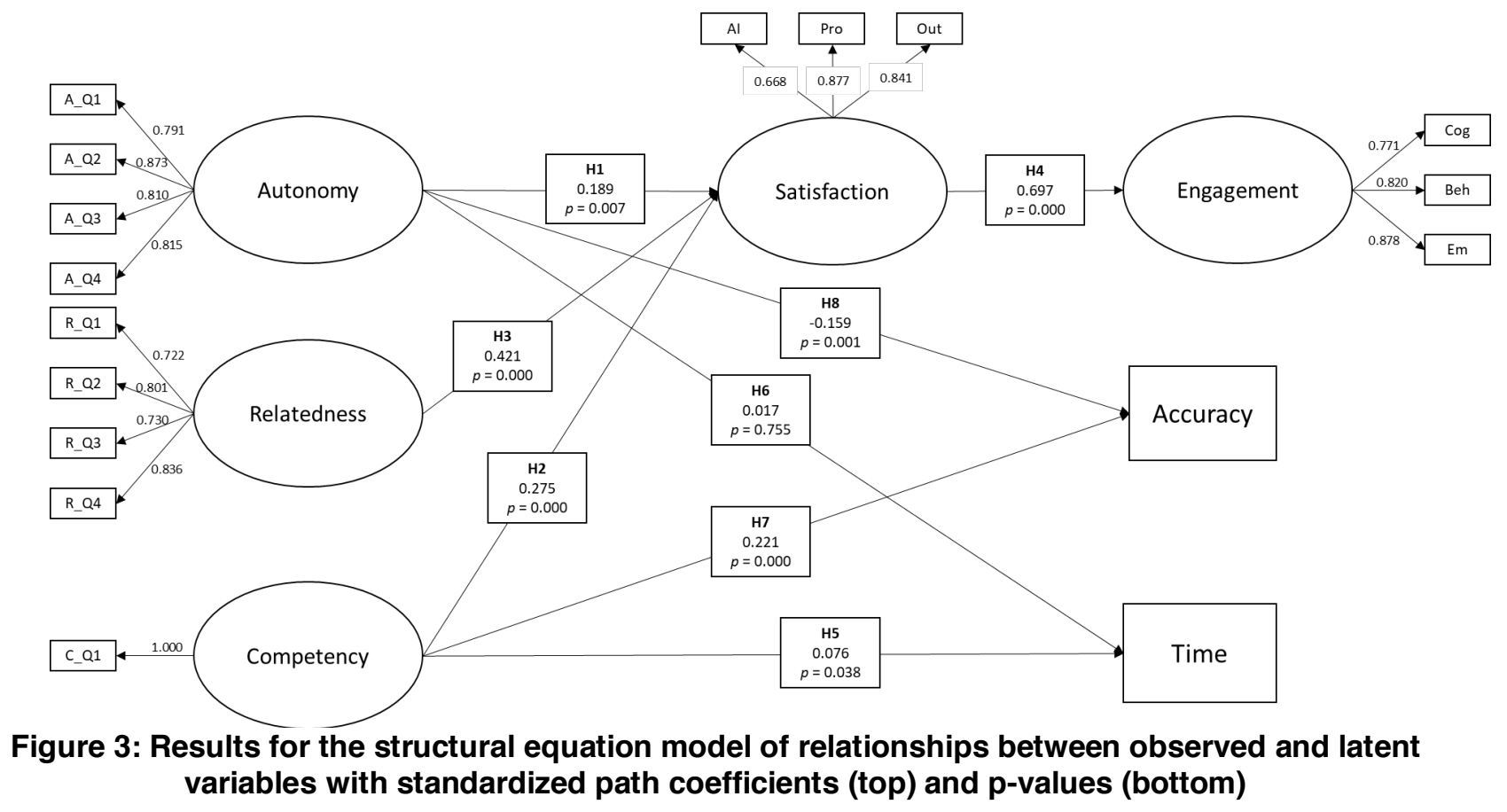

$\chi^{2}(105)=372.99$, RMSEA $[90 \% \mathrm{CI}]=0.078[0.069$, $0.086]$, CFI $=0.847$, TLI $=0.802$, $\mathrm{SRMR}=0.057$. As expected, satisfaction emerged as a mediator between the SDT needs and engagement with the bot.

$\mathrm{H} 1, \mathrm{H} 2$, and $\mathrm{H} 3$ predicted that perceived autonomy, competency, and relatedness regarding the chatbot interaction should be related to the perceived satisfaction with the chatbot. As shown in Figure 2, the results do support all three hypotheses $\left(\gamma_{1}=0.189, \mathrm{SE}=\right.$ $0.046, p<0.01 ; \gamma_{2}=0.275, \mathrm{SE}=0.038, p<0.01 ; \gamma_{3}=$ $0.421, \mathrm{SE}=0.072, p<0.01$ ).

$\mathrm{H} 4$ predicted that satisfaction with the chatbot would be related to greater engagement with the interaction. This hypothesis was also supported by the results $\left(\gamma_{4}=0.697, \mathrm{SE}=0.109, p<0.01\right)$.

$\mathrm{H} 5$ and $\mathrm{H} 6$ predicted that perceived competency would be positively related and perceived autonomy would be negatively related to efficiency of decisionmaking. These hypotheses were not supported by the results. Counter to our intuitions, greater competency was seen to be marginally related to an increase in time taken, and hence lower efficiency, when completing the task ( $\gamma_{5}$ $=0.076, \mathrm{SE}=0.443, p<0.05)$. While these small numbers should be interpreted with caution, it is possible to assume that this could have resulted from the greater number of clicks participants had to make in the higher SDT needs conditions, which is a limitation of this study. In addition, there was no significant relationship between autonomy and time taken to complete the task $\left(\gamma_{6}=0.017\right.$, $\mathrm{SE}=0.531, p=0.76$ ).

Lastly, $\mathrm{H} 7$ and $\mathrm{H} 8$ predicted that perceived competency would be positively related and perceived

autonomy would be negatively related to accuracy of decision-making. Both of these hypotheses were supported by the results $\left(\gamma_{7}=0.221, \mathrm{SE}=0.086, p<0.01\right.$; $\left.\gamma_{8}=-0.159, \mathrm{SE}=0.073, p<0.01\right)$.

\section{Discussion \& Conclusions}

In this study we explored the effect of the three needs as described by SDT (competency, autonomy, relatedness) in terms of their association with user satisfaction, user engagement, decision-making accuracy, and decision-making efficiency when users are being assisted by a chatbot to make decisions. Our carefully designed experiment provides compelling evidence that virtual agents that satisfy these three needs are significantly related to user engagement and user satisfaction. Moreover, we found that perceptions of autonomy are negatively related to decision accuracy and to decision efficiency. Finally, we found that perceptions of competence are positively related to accuracy but are negatively related to efficiency.

\subsection{Theoretical implications}

This study has a number of theoretical contributions. First, our research highlights the importance of considering psychological constructs during AI design. Appropriate AI design is critical to establish a relationship of trust and dependability with the AI [59]. Yet, both researchers and practitioners have placed disproportionate attention to studying and capturing the 
functionality of AI design without much effort spent towards assessing the impact on these designs on individuals. This study aims to address this gap by infusing psychological constructs into the AI development starting from the design phase.

Second, this study deepens our understanding of SDT in the area of AI assisted decision-making. We found that achieving each of the three motivational needs results in an increased sense of satisfaction with the AI, with the decision-making process, and with the outcome of the decision. We also found that the three needs have a direct and indirect effect on how intensely users engage with the AI assistant and use it for their decision-making. Finally, our study demonstrates the interesting role of sense of competency and sense of autonomy in the decision speed and accuracy. In alignment with the literature, our study demonstrates that the negative relationship between autonomy and accuracy persists even with the use of AI. Moreover, the study reveals an interesting relationship between sense of competency and decision speed. In alignment with the mixed findings in the literature, the pattern seems to be obtuse between these two constructs. It reflects the need for more research to parse the circumstances under which competency leads to efficiency and circumstances under which it does not.

\subsection{Practical Implications}

Our findings also offer useful implications for AI designers and organizations that plan on using AI assisted chatbots to improve decision-making efforts. For instance, AI designers can create the designs such that users can relate more to the $\mathrm{AI}$ and thus achieve more meaningful outcomes. In addition, designers can create user friendly designs such that the AI makes the user feel competent in understanding and solving the problem. Finally, the AI designers can allow enough sense of autonomy within the users such that they feel sufficiently in control of the situation to engage with the AI wholeheartedly without fear of losing control.

This study is also useful for organizations by giving them an insight into the design of AI assisted chatbots such that these tools achieve the desired goals. Even when the design considerations cannot accommodate optimal levels of motivational needs, organizations can establish adequate trainings and afford choices to the users such that the users feel competent and in control of their relationship with the AI and feel that the AI is present to enrich their decision-making rather than replace their position at the organization.

\subsection{Limitations and Future Research}

There are several limitations that have to be considered when interpreting the findings of this study.
Each of these limitations, however, offers exciting opportunities for future research. First, our study examined the role of SDT in AI design, but the AI was a simulated environment with no dynamic responses. That may have lessened the sophisticated nature of the AI interaction and thus influenced user behaviors. In the future, it would be worthwhile to use a more advanced AI environment to replicate the study.

Second, the study could not successfully manipulate the competency condition. Users in both high and low competency conditions appeared to feel not competent enough to solve the problem. While competency did have significant effect on the variables of interest, these results should be interpreted with caution. In the future, it may be useful to utilize a decision-making activity that the users could be appropriately trained in. Using actual decision-making scenarios that the users face in their life may also yield different results.

Finally, the study used a Logigram puzzle as the decision-making activity. This requires solving a puzzle from the hints provided. These hints were provided by the AI. Since these hints were originally meant to be read independently, the presence of the AI may not have made much of a difference. In the future, it would be useful to have a decision-making activity where the AI interactions are more sophisticated.

\section{References}

[1] Araujo, T.: 'Living up to the chatbot hype: The influence of anthropomorphic design cues and communicative agency framing on conversational agent and company perceptions', Computers in Human Behavior, 2018, 85, pp. 183-189

[2] Arsenijevic, U., and Jovic, M.: 'Artificial Intelligence Marketing: Chatbots', in: 'Artificial Intelligence Marketing: Chatbots’ (IEEE, 2019, edn.), pp. 19-193

[3]] Atre, J.A.: 'Choice under conditions of abundance: The behavior of television audiences', University of Pennsylvania, 2007

[4] Balicer, R.D., and Cohen-Stavi, C.: 'Advancing Healthcare Through Data-Driven Medicine and Artificial Intelligence': 'Healthcare and Artificial Intelligence' (Springer, 2020), pp. 9-15

[5] Bennett, C.C., and Hauser, K.: 'Artificial intelligence framework for simulating clinical decision-making: A Markov decision process approach', Artificial intelligence in medicine, 2013, 57, (1), pp. 9-19

[6] Briggs, R.O., Reinig, B.A., and de Vreede, G.-J.: 'Meeting satisfaction for technology-supported groups: An empirical validation of a goal-attainment model', Small Group Research, 2006, 37, (6), pp. 585-611

[7] Briggs, R.O., Reinig, B.A., and de Vreede, G.-J.: 'The yield shift theory of satisfaction and its application to the IS/IT domain', Journal of the Association for Information Systems, 2008, 9, (5), pp. 14

[8] Carțiş, A.-I., and Suciu, D.M.: 'Chatbots as a Job Candidate Evaluation Tool', in: 'Chatbots as a Job Candidate Evaluation Tool' (Springer, 2019, edn.), pp. 189-193 
[9] Chaves, A.P., and Gerosa, M.A.: 'How should my chatbot interact? A survey on human-chatbot interaction design', arXiv preprint arXiv: 1904.02743, 2019

[10] Chaves, A.P., and Gerosa, M.A.: 'Single or Multiple Conversational Agents? An Interactional Coherence Comparison', in: 'Single or Multiple Conversational Agents? An Interactional Coherence Comparison' (2018, edn.), pp. 1-13

[11] Davis, D.A., Mazmanian, P.E., Fordis, M., Van Harrison, R., Thorpe, K.E., and Perrier, L.: 'Accuracy of Physician Self-assessment Compared With Observed Measures of CompetenceA Systematic Review', JAMA, 2006, 296, (9), pp. 1094-1102

[12] de Vreede, T., Andel, S., de Vreede, G.-J., Spector, P., Singh, V., and Padmanabhan, B.: 'What is Engagement and How Do We Measure It? Toward a Domain Independent Definition and Scale', in: 'What is Engagement and How Do We Measure It? Toward a Domain Independent Definition and Scale' (IEEE Computer Society Press, 2019, edn.), pp.

[13] Deci, E.L., and Ryan, R.M.: 'Self-determination and intrinsic motivation in human behavior', EL Deci, RM Ryan.-1985, 1985

[14] Dennis, A.R., and Carte, T.A.: 'Using geographical information systems for decision making: Extending cognitive fit theory to map-based presentations', Information Systems Research, 1998, 9, (2), pp. 194-203

[15] Duffy, B.: 'Anthropomorphism and the social robot', Robotics and autonomous systems, 2003, 42, (3-4), 177-190

[16] Duijst, D. 'Can we improve the user experience of chatbots with personalisation', MS thesis. Univ. of Amsterdam, 2017

[17] Esteva, A., Kuprel, B., Novoa, R.A., Ko, J., Swetter, S.M., Blau, H.M., and Thrun, S.: 'Dermatologist-level classification of skin cancer with deep neural networks', nature, 2017, 542, (7639), pp. 115-118

[18] Fethi, M.D., and Pasiouras, F.: 'Assessing bank efficiency and performance with operational research and artificial intelligence techniques: A survey', European journal of operational research, 2010, 204, (2), pp. 189-198

[19] Frommert, C., Häfner, A., Friedrich, J., and Zinke, C.: 'Using chatbots to assist communication in collaborative networks', in: 'Using chatbots to assist communication in collaborative networks' (Springer, 2018, edn.), pp. 257-265

[20] Goleman, D.: 'Emotional Intelligence, why it Can Matter More Than IQ; \& Working with Emotional Intelligence' (Bloomsbury, 1996. 1996)

[21] Hepler, T.J., and Chase, M.A.: 'Relationship between decision-making self-efficacy, task self-efficacy, and the performance of a sport skill', Journal of Sports Sciences, 2008, 26, (6), pp. 603-610

[22] Hepler, T.J., and Feltz, D.L.: 'Path analysis examining selfefficacy and decision-making performance on a simulated baseball task', Research quarterly for exercise and sport, 2012, 83, (1), pp. 55-64

[23] Hepler, T.J., and Feltz, D.L.: 'Take the first heuristic, selfefficacy, and decision-making in sport', Journal of Experimental Psychology: Applied, 2012, 18, (2), pp. 154

[24] Horváth, D.: 'From user feedback to requirements using chatbots', 2019

[25] Huang, L.: 'Qualitative analysis of the application of selfdetermination theory in robotics tournaments', in:
'Qualitative analysis of the application of selfdetermination theory in robotics tournaments' (2017, edn.), pp. $135-136$

[26] Huang, M.-H., and Rust, R.T.: 'Artificial intelligence in service', Journal of Service Research, 2018, 21, (2), pp. $155-172$

[27] Huang, M.-H., and Rust, R.T.: 'Engaged to a Robot? The Role of AI in Service', Journal of Service Research, 2020, pp. 1094670520902266

[28] Hung, S.Y., and Liang, T.P.: 'Effect of computer selfefficacy on the use of executive support systems', Industrial Management \& Data Systems, 2001

[29] Jain, M., Kumar, P., Kota, R., and Patel, S.N.: 'Evaluating and informing the design of chatbots', in: 'Evaluating and informing the design of chatbots' (2018, edn.), pp. 895-906

[30] John Bernardin, H., Thomason, S., Ronald Buckley, M., and Kane, J.S.: 'Rater rating-level bias and accuracy in performance appraisals: The impact of rater personality, performance management competence, and rater accountability', Human Resource Management, 2016, 55, (2), pp. 321-340

[31] Johnson, C.H.: 'Reflections on leadership', naval war college review, 2014, 67, (1), pp. 135-144

[32] Kim, Y.H., Kim, D.J., Wachter, K.: 'A study of mobile user engagement (MoEN): Engagement motivations, perceived value, satisfaction, and continued engagement intention', Decision support systems, 2013, 56, pp. 361-370

[33] La Guardia, J.G., Ryan, R.M., Couchman, C.E., and Deci, E.L.: 'Within-person variation in security of attachment: A self-determination theory perspective on attachment, need fulfillment, and well-being', Journal of personality and social psychology, 2000, 79, (3), pp. 367

[34] Langenkamp, M., Costa, A., and Cheung, C.: 'Hiring Fairly in the Age of Algorithms', arXiv preprint arXiv:2004.07132, 2020

[35] Leachman, S.A., and Merlino, G.: 'Medicine: The final frontier in cancer diagnosis', Nature, 2017, 542, (7639), pp. 36-38

[36] Lenton, A.P., Fasolo, B., and Todd, P.M.: 'Who is in your shopping cart? Expected and experienced effects of choice abundance in the online dating context': 'Evolutionary psychology and information systems research' (Springer, 2010), pp. 149-167

[37] Liao, Q.V., Mas-ud Hussain, M., Chandar, P., Davis, M., Khazaeni, Y., Crasso, M.P., Wang, D., Muller, M., Shami, N.S., and Geyer, W.: 'All Work and No Play?', in 'All Work and No Play?' (2018, edn.), pp. 1-13

[38] Luger, E., and Sellen, A.: " Like Having a Really Bad PA" The Gulf between User Expectation and Experience of Conversational Agents', in: "Like Having a Really Bad PA" The Gulf between User Expectation and Experience of Conversational Agents' (2016, edn.), pp. 5286-5297

[39] Luo, X., Tong, S., Fang, Z., and Qu, Z.: 'Frontiers: Machines vs. humans: The impact of artificial intelligence chatbot disclosure on customer purchases', Marketing Science, 2019, 38, (6), pp. 937-947

[40] Madakam, S., Holmukhe, R.M., and Jaiswal, D.K.: 'The future digital work force: Robotic process automation (RPA)', JISTEM-Journal of Information Systems and Technology Management, 2019, 16 
[41] Menard, P., Bott, G.J., and Crossler, R.E.: 'User motivations in protecting information security: Protection motivation theory versus self-determination theory', Journal of Management Information Systems, 2017, 34, (4), pp. 1203-1230

[42] Mîndrilã, D.: 'Maximum likelihood (ML) and diagonally weighted least squares (DWLS) estimation procedures: A comparison of estimation bias with ordinal and multivariate non-normal data', International Journal of Digital Society, 2010, 1, (1), pp. 60-66

[43] Morana, S., Gnewuch, U., Jung, D., and Granig, C.: 'The Effect of Anthropomorphism on Investment DecisionMaking with Robo-Advisor Chatbots', in: 'The Effect of Anthropomorphism on Investment Decision-Making with Robo-Advisor Chatbots' (2020, edn.), pp.

[44] Neys, J.L., Jansz, J., Tan, E.S.: 'Exploring persistence in gaming: The role of self-determination and social identity', Computers in Human Behavior, 2014, 37, pp. 196-209

[45] Nguyen, Q.N., and Sidorova, A.: 'Understanding user interactions with a chatbot: A self-determination theory approach', 2018

[46] Oyewole, O.: 'Conversational design: the use of chatbot as an electoral information dissemination tool', 2020

[47] Parker, A.M., and Fischhoff, B.: 'Decision-making competence: External validation through an individualdifferences approach', Journal of Behavioral Decision Making, 2005, 18, (1), pp. 1-27

[48] Patel, V.L., Shortliffe, E.H., Stefanelli, M., Szolovits, P., Berthold, M.R., Bellazzi, R., and Abu-Hanna, A.: 'The coming of age of artificial intelligence in medicine', Artificial intelligence in medicine, 2009, 46, (1), pp. 5-17

[49] Rasch, R., Kott, A., and Forbus, K.D.: 'Incorporating AI into military decision making: an experiment', IEEE Intelligent Systems, 2003, 18, (4), pp. 18-26

[50] Ren, R., Castro, J.W., Acuña, S.T., and de Lara, J.: 'Usability of Chatbots: A Systematic Mapping Study', in: 'Usability of Chatbots: A Systematic Mapping Study' (2019, edn.), pp. 479-617

[51] Rezvani, A., Khosravi, P., and Dong, L.: 'Motivating users toward continued usage of information systems: Selfdetermination theory perspective', Computers in Human Behavior, 2017, 76, pp. 263-275

[52] Roca, J.C., and Gagné, M.: 'Understanding e-learning continuance intention in the workplace: A selfdetermination theory perspective', Computers in human behavior, 2008, 24, (4), pp. 1585-1604

[53] Rosseel, Y.: 'Lavaan: An R package for structural equation modeling and more. Version 0.5-12 (BETA)', Journal of statistical software, 2012, 48, (2), pp. 1-36

[54] Ryan, R.M., Deci, E.L.: 'Self-determination theory and the facilitation of intrinsic motivation, social development, and well-being', American psychologist, 2000, 55, (1), pp. 68

[55] Sabharwal, N., and Agrawal, A.: 'New Research in the Field of Cognitive Virtual Chatbots': 'Cognitive Virtual Assistants Using Google Dialogflow’ (Springer, 2020), pp. 183-186

[56] Santosa, P.I., Wei, K.K., and Chan, H.C.: 'User involvement and user satisfaction with information-seeking activity', European Journal of Information Systems, 2005, 14, (4), pp. 361-370
[57] Shanmuganathan, M.: 'Behavioural finance in an era of artificial intelligence: Longitudinal case study of roboadvisors in investment decisions', Journal of Behavioral and Experimental Finance, 2020, pp. 100297

[58] Shawar, B., Atwell, E.: 'Chatbots: are they really useful?', in: 'Chatbots: are they really useful?' (2007, edn.), 29-49

[59] Siau, K., and Wang, W.: 'Building trust in artificial intelligence, machine learning, and robotics', Cutter Business Technology Journal, 2018, 31, (2), pp. 47-53

[60] Sironi, P.: 'FinTech innovation: from robo-advisors to goal based investing and gamification' (John Wiley, 2016)

[61] Sørebø, Ø., Halvari, H., Gulli, V.F., Kristiansen, R.: 'The role of self-determination theory in explaining teachers' motivation to continue to use e-learning technology', Computers \& Education, 2009, 53, (4), pp. 1177-1187

[62] Steckel, J.H., Winer, R.S., Bucklin, R.E., Dellaert, B.G., Drèze, X., Häubl, G., Jap, S.D., Little, J.D., Meyvis, T., and Montgomery, A.L.: 'Choice in interactive environments', Marketing Letters, 2005, 16, (3-4), pp. 309-320

[63] Steels, L., and Brooks, R.: 'The artificial life route to artificial intelligence: Building embodied, situated agents' (Routledge, 2018. 2018)

[64] Sternberg, R.J.: 'The theory of successful intelligence', Interamerican Journal of Psychology, 2005, 39, (2), pp. 189-202

[65] Sternberg, R.J.: 'Toward a triarchic theory of human intelligence', Behavioral and Brain Sciences, 1984, 7, (2), pp. 269-287

[66] Suh, A., Wagner, C., and Liu, L.: 'Enhancing user engagement through gamification', Journal of Computer Information Systems, 2018, 58, (3), pp. 204-213

[67] Team, R.C.: 'R: A language and environment for statistical computing', in: 'R: A language and environment for statistical computing' (Vienna, Austria, 2013, edn.), pp.

[68] Trivedi, J.: 'Examining the customer experience of using banking Chatbots and its impact on brand love: the moderating role of perceived risk', Journal of internet Commerce, 2019, 18, (1), pp. 91-111

[69] Turing, A.: 'Computing machinery and intelligence, Mind LIX, 433-60', in: 'Computing machinery and intelligence, Mind LIX, 433-60’ (1950, edn.), pp.

[70] Wakabayashi, D.: 'Self-driving Uber car kills pedestrian in Arizona, where robots roam', New York Times, 2018, 19

[71] Wallace, R.S.: 'The anatomy of ALICE': 'Parsing the Turing Test' (Springer, 2009), pp. 181-210

[72] Weizenbaum, J.: 'ELIZA - a computer program for the study of natural language communication between man and machine', Communications of the ACM, 1966, 9, (1), 36-45

[73] Witwicki, S., Castillo, J.C., Messias, J., Capitan, J., Melo, F.S., Lima, P.U., and Veloso, M.: 'Autonomous surveillance robots: A decision-making framework for networked muiltiagent systems', IEEE Robotics \& Automation Magazine, 2017, 24, (3), pp. 52-64

[74] Zamora, J.: 'Rise of the chatbots: Finding a place for artificial intelligence in India and US', in: 'Rise of the chatbots: Finding a place for artificial intelligence in India and US' (2017, edn.), pp. 109-112

[75] Zumstein, D., and Hundertmark, S.: 'Chatbots - An Interactive Technology for Personalized Communication transactions and services', IADIS International Journal on WWW/Internet, 2017, 15, (1) 